\title{
Home enteral nutrition in children-2010 nationwide survey of the polish society for clinical nutrition of children
}

\author{
Agnieszka Szlagatys-Sidorkiewicz • \\ Katarzyna Popińska • Ewa Toporowska-Kowalska • \\ Anna Borkowska • Marta Sibilska • \\ Beata Gębora-Kowalska • Stanisław Kłęk • \\ Ewa Hapyn • Jarosław Kierkuś • \\ Urszula Grzybowska-Chlebowczyk • Sabina Więcek • \\ Adam Daukszewicz • Marlena Jakubczyk • \\ Mariola Lembas-Sznabel • Marek Wilczyński • \\ Ilona Zagożdżon • Przemysław Matras • \\ Anna Zmarzly • Janusz Książyk
}

Received: 22 November 2011 / Accepted: 29 November 2011 /Published online: 15 December 2011

(C) The Author(s) 2011. This article is published with open access at Springerlink.com

\begin{abstract}
Published epidemiologic data on the administration rates of enteral/parenteral home nutrition is very limited. The aim of this first nationwide study was to assess the availability of pediatric home enteral nutrition (HEN) services in Poland.
\end{abstract}

A. Szlagatys-Sidorkiewicz $(\bowtie) \cdot$ A. Borkowska

Department of Pediatrics, Gastroenterology,

Hepatology and Nutrition, Medical University of Gdansk,

ul. Nowe Ogrody 1-6,

80-03, Gdansk, Poland

e-mail: aga1@gumed.edu.pl

K. Popińska $\cdot$ M. Sibilska $\cdot$ J. Książyk

Department of Pediatrics,

The Children's Memorial Health Institute,

Warsaw, Poland

E. Toporowska-Kowalska • B. Gębora-Kowalska

Department of Allergology, Gastroenterology and Nutrition,

Medical University of Lodz,

Lodz, Poland

\section{S. Kłęk}

Medical Center Nutrimed,

Cracow, Poland

E. Hapyn

Department of Pediatrics and Gastroenterology,

Area Hospital in Torun,

Torun, Poland

J. Kierkuś

Department of Gastroenterology, Hepatology and Immunology,

The Children's Memorial Institute,

Warsaw, Poland
The questionnaire was sent to all regional centers providing pediatric HEN services in Poland $(n=14)$. The analysis included the number of pediatric patients who received HEN in 2010, their demographic characteristics and geographical

U. Grzybowska-Chlebowczyk $\cdot$ S. Więcek

Department of Pediatrics, Silesian Medical Academy,

Katowice, Poland

\section{A. Daukszewicz}

Gastroenterology Clinic, Children's Hospital,

Olsztyn, Poland

M. Jakubczyk

Clinic for Home Enteral and Parenteral Nutrition,

Department of Anesthesiology and Intensive Therapy,

Collegium Medicum of Bydgoszcz,

Nicolaus Copernicus University in Torun,

Torun, Poland

M. Lembas-Sznabel

Center for Palliative Care,

Szczecin, Poland

M. Wilczyński

Ars Medica Health Center,

Krotoszyn, Poland

\section{Zagożdżon}

Department of Nephrology and Hypertension,

Medical University of Gdansk,

Gdansk, Poland 
distribution. Furthermore, the distributions of indications and methods of enteral nutrition administration were analyzed, along with the reasons of withdrawal from the HEN program. The number and fraction of children receiving HEN increased in 2010, from 433 (11.34 per 1 million inhabitants) on January 1st to 525 (13.75) on December 31st. Marked differences were observed in geographical distribution of this parameter, from zero to up to 30 pediatric patients per 1 million inhabitants. Median age of patients was 6 years (range: 9 months18 years). In most cases, HEN was prescribed due to neurological disorders $(n=337,64.2 \%)$, and administered by means of gastrostomy $(n=450,85.71 \%)$. This study revealed the dynamic development of pediatric HEN services in Poland but also documented their potential regional shortages.

Keywords Children · Gastrostomy · Home enteral nutrition · Jejunostomy

\section{Introduction}

Recent years were associated with substantial development of enteral nutrition, and particularly, home enteral nutrition (HEN) [14]. For the last ten to 20 years, HEN has been fully reimbursed in many European countries which has contributed to increased popularity of this treatment modality $[8$, 13]. Also, in Poland, HEN has been developed and is reimbursed by the National Health Fund (NHF) since 2007. According to the regulations of NHF, however, enteral feeding is reimbursed only if there is no possibility of oral nutrition, which excludes oral supplementation or sip feeding included in the ESPEN definition of enteral feeding [11].

Enteral nutrition is required in all patients who are unable to cover their energetic and nutritional requirements by means of normal ingestion despite, at least partially, retained function of the gastrointestinal tract. Potential advantages of HEN in comparison to traditional hospital-based enteral nutrition include shorter hospitalization, lower direct costs of therapy and lower risk of secondary malnutrition-associated complications.

According to sparse literature data, children constitute a minority among patients receiving HEN [15]. Published epidemiologic data on the administration rates of enteral/ parenteral home nutrition is very limited and available only

\section{P. Matras}

1st Department of Surgery, Transplantology and Nutrition,

Medical University in Lublin,

Lublin, Poland

A. Zmarzly

Home Enteral Nutrition Clinic, Area Hospital,

Wroclaw, Poland in a few countries $[2,3,9,15]$. In turn, reliable data on the administration rates of HEN is required to assess potential benefits associated with this treatment modality along with types and rates of associated complications. Additionally, it can be helpful in confronting requirements for this type of procedure with available resources. Finally, actual data is necessary for planning prospective studies of therapeutic outcomes of enteral nutrition. Constantly updated registries constitute the most optimal source of reliable data on HEN service availability and utilization rates. Despite their obvious advantages, only a few such national registries exist, however $[2,3,7,15]$.

Since it was reimbursed and thus available, the dynamic increase in the number of patients who were offered HEN services by Polish medical centers was observed during recent years. However, still there are some regions with no specialized centers offering HEN services for children. Therefore, the aim of this first nationwide study was to assess the availability of pediatric HEN services in Poland in order to identify potential areas that should be improved.

\section{Materials and methods}

This study was based on a retrospective 1-year analysis of HEN services that were offered to Polish children in 2010. All public healthcare services in Poland are covered by the regional branches of the same funder, National Health Fund. Therefore, all 16 Polish provinces have the same HEN reimbursement criteria and procedures. According to these regulations, each healthcare provider is responsible for qualifying patients to nutritional treatment, training patients (or their parents - in the case of underage subjects) in HEN procedures, providing diets and all related devices, followup visits and all other necessary medical services.

In January 2010, the questionnaire, developed by the Polish Society for Clinical Nutrition of Children and previously tested for reliability and validity, was sent to all regional centers providing pediatric HEN services in Poland $(n=14)$. In each center, one person was assigned the responsibility of data collection. All participating centers returned completed questionnaires between January and March of 2011.

The results of the survey were analyzed with an aid of Statistica 8 (StatSoft $\left.{ }^{\circledR}\right)$ package. The analysis included the number of pediatric patients who received HEN on January 1st 2010 and December 31st 2010, their demographic characteristics and geographical distribution. The prevalence rate of HEN was expressed as the number of children receiving this type of service per one 1 million of the inhabitants in the analyzed region. Furthermore, the distributions of indications and methods of enteral nutrition administration were analyzed, along with the potential reasons of withdrawal from the HEN program. 
Table 1 Exclusion criteria from polish pediatric HEN program in 2010

\begin{tabular}{ll}
\hline Criterion & Number \\
\hline Lack of satisfactory outcome & 1 \\
Complications associated with enteral nutrition & 2 \\
Lack of patient's acceptance & 7 \\
Lack of caregivers' acceptance & 7 \\
Satisfactory weight gain & 20 \\
Patient's death & 17 \\
Other & 19 \\
Total & 73 \\
\hline
\end{tabular}

\section{Results}

The number of children receiving HEN in 2010 increased by $21 \%$ (from 433 to 525 patients) compared to January 1 st 2010. Within the study period, 73 patients were withdrawn from the HEN program due to various reasons (Table 1), and 165 new subjects were qualified to the enteral feeding (31.43\%).

The fraction of children receiving HEN increased substantially in 2010 , from 11.34 per 1 million inhabitants on January 1st to 13.75 per 1 million on December 31 st. Marked differences were observed in terms of geographical distribution of this parameter, from zero (in two provinces) to up to 30 pediatric patients per 1 million inhabitants (Fig. 1).

Most patients receiving HEN ( $n=419,79.8 \%)$ were supervised by specialized pediatric centers, while the remainder $(n=106,20.2 \%)$ were handled by medical centers serving both children and adults.

Median age of patients receiving HEN in 2010 was 6 years (range: 9 months-18 years). This group included 247 girls

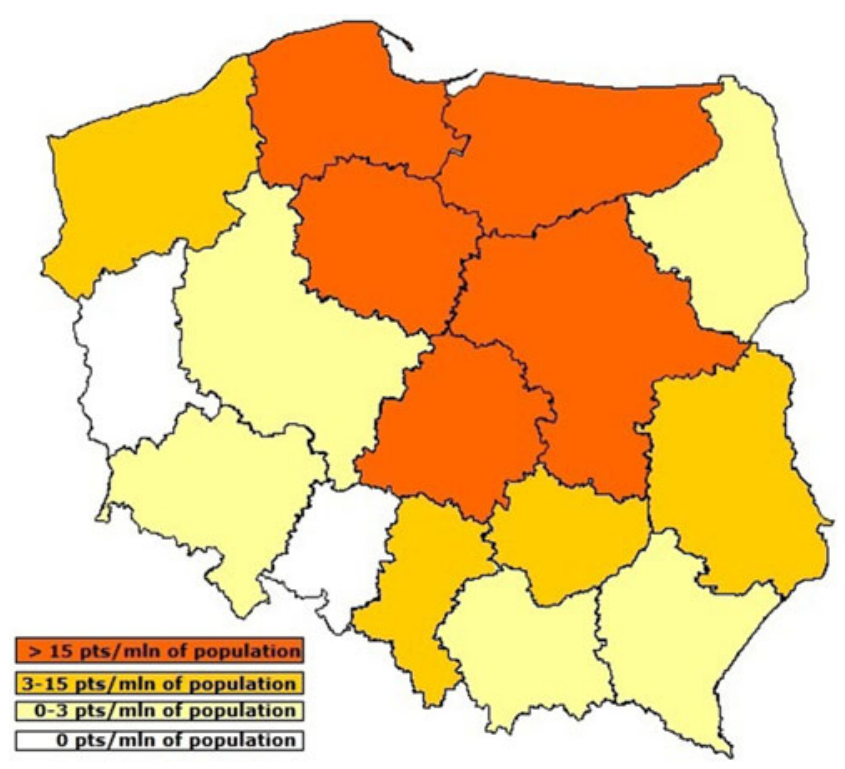

Fig. 1 Geographic distribution of polish children receiving HEN in 2010
Table 2 Indications for enteral nutrition in polish children receiving HEN in 2010

\begin{tabular}{llr}
\hline Indication & Number & Percentage \\
\hline Cerebral palsy & 164 & 31.2 \\
Encephalopathy & 52 & 9.9 \\
Spinal muscular atrophy (SMA) & 44 & 8.4 \\
Muscular dystrophy & 6 & 1.1 \\
Other neurological disorders & 71 & 13.5 \\
Genetic syndromes & 93 & 17.7 \\
Short bowel syndrome & 1 & 0.2 \\
Inflammatory bowel disease & 3 & 0.6 \\
Other gastrointestinal diseases & 26 & 5.0 \\
Cystic fibrosis & 20 & 3.8 \\
Chronic renal failure & 15 & 2.9 \\
Chronic liver failure & 1 & 0.2 \\
Oncological diseases & 5 & 1.0 \\
Malformation syndrome & 11 & 2.1 \\
Congenital heart disease & 4 & 0.8 \\
Metabolic disorders & 9 & 1.7 \\
Total & 525 & 100.0 \\
\hline
\end{tabular}

(47.5\%) and 278 boys (52.5\%). In most cases, HEN was prescribed due to neurological disorders $(n=337,64.2 \%)$. Other indications for HEN are summarized in Table 2. In most cases $(n=450,85.71 \%)$, enteral nutrition was administered by means of gastrostomy (Table 3).

\section{Discussion}

This first Polish nationwide survey on HEN prevalence rates among pediatric patients included data from all centers offering this type of specialized services. Throughout 2010, a substantial increase was observed in the number of HEN-receiving children. Nonetheless, the fraction of Polish pediatric patients receiving HEN (13.75 per 1 million inhabitants) was still markedly lower compared to published data from other countries: 48.23 cases per 1 million in some regions of Italy [15] and 95.6 per 1 million in the United

Table 3 Methods of enteral nutrition administration in polish children receiving HEN in 2010

\begin{tabular}{llc}
\hline Method & Number & Percentage \\
\hline Nasogastric tube & 59 & 11.2 \\
Gastrostomy & 450 & 85.7 \\
Nasojejunal tube & 2 & 0.4 \\
Jejunostomy & 4 & 0.8 \\
Gastrojejunal tube & 10 & 1.90 \\
Total & 525 & 100.0 \\
\hline
\end{tabular}


Kingdom [1]. It should be remembered, however, that 2010 corresponded to already the fourth year of pediatric HEN reimbursement/availability in Poland. One can assume this as one potential reason for both relatively low prevalence of HEN services and dynamic increase in prescription rates of enteral feeding.

Another important finding of this study is uneven geographic distribution of HEN administration rates. Home enteral nutrition was not prescribed to children living in two out of 16 provinces, probably due to the lack of centers offering HEN services in these administrative regions of Poland. In another five provinces, the rates of HEN administration were lower than three per 1 million inhabitants. However, there were several provinces where markedly higher HEN administration rates (above 15 per 1 million inhabitants) were reported. Similar regional differences in HEN distribution in children were previously reported from Spain and Italy $[3,15]$. One should confer this highly variable HEN administration rates in Poland to the prevalence rates of pediatric disorders that usually require enteral nutrition in respective regions. Any discrepancies between these two measures would suggest potential regional deficiency of HEN services and eventually substantiate funding new pediatric centers.

Our survey documented that neurologic disorders constituted principle indication for enteral nutrition in Polish children (64.2\%). Also, in other European countries, chronic neurologic disorders predominate among indications for HEN, but the fractions of pediatric patients receiving this treatment modality due to neurological reasons are lower: $28.23 \%$ in Spain [7], 35\% in France [4], and 50\% in Italy [15]. Interestingly, 11-year retrospective analysis by Daveluy et al. [5] revealed substantial change in the distribution of indications for HEN administered in French children. In 2000, chronic neurologic disorders constituted the most frequent indication for HEN, in contrast to 1989 when enteral nutrition was usually prescribed due to primary alimentary disorders [5].

Compared to other European countries, our study revealed markedly lower percentage of pediatric patients who received HEN due to respiratory and circulatory tract disorders, and chronic kidney or liver failure. Perhaps this underrepresentation of indications other than neurological and alimentary disorders resulted from recent educational activities of the Polish Society for Clinical Nutrition of Children. During our certified courses, we focused on prescribing HEN in chronic neurological and alimentary conditions. The results of this study suggest, however, that during future courses provided by the Society more attention should be paid to other disorders that may potentially require enteral nutrition.

Recent evidence suggests that cystic fibrosis is a condition which definitely can benefit from enteral nutrition $[6$, $10,12,16,17]$. In one study, cystic fibrosis patients corresponded to $23 \%$ of pediatric patients receiving enteral nutrition in France [4]. In contrast, our survey revealed only $20(3.6 \%)$ cystic fibrosis cases among children who were offered HEN in 2010. Taking into account rough estimates of cystic fibrosis prevalence in Poland $(n=1,500)$, enteral nutrition was prescribed to only $1.3 \%$ of this group, hence to six-fold lower fraction compared to tube-feed cystic fibrosis cases managed in the United States.

Open Access This article is distributed under the terms of the Creative Commons Attribution Noncommercial License which permits any noncommercial use, distribution, and reproduction in any medium, provided the original author(s) and source are credited.

\section{References}

1. Baxter J (2010) Paediatric home parenteral nutrition (HPN). In: Smith T, Micklewright A, Hirst A, Gowan H, Bakter J (eds) Annual BANS Report, 2010. Artificial nutrition support in the UK 2000-2009. A report by the British Artificial Nutrition Survey (BANS), a committee of BAPEN (The British Association for Parenteral and Enteral Nutrition. BAPEN, Worcs, pp 49-52

2. Beath SV, Gowen H, Puntis JW (2011) Trends in paediatric home parenteral nutrition and implications for service development. Clin Nutr 30(4):499-502

3. Cuerda C, Planas M, Gomez Candela C, Luengo LM (2009) Trends in home enteral nutrition in Spain: analysis of the NADYA registry 1992-2007. Nutr Hosp 24(3):347-353

4. Daveluy W, Guimber D, Mention K, Lescut D, Michaud L, Turck D, Gottrand F (2005) Home enteral nutrition in children: an 11year experience with 416 patients. Clin Nutr 24(1):48-54

5. Daveluy W, Guimber D, Uhlen S, Lescut D, Michaud L, Turck D, Gottrand F (2006) Dramatic changes in home-based enteral nutrition practices in children during an 11-year period. J Pediatr Gastroenterol Nutr 43(2):240-244

6. Efrati O, Mei-Zahav M, Rivlin J, Kerem E, Blau H, Barak A, Bujanover Y, Augarten A, Cochavi B, Yahav Y, Modan-Moses D (2006) Long term nutritional rehabilitation by gastrostomy in Israeli patients with cystic fibrosis: clinical outcome in advanced pulmonary disease. J Pediatr Gastroenterol Nutr 42(2):222-228

7. Gomez-Lopez L, Martinez-Costa C, Pedron-Giner C, CalderonGarrido C, Navas Lopez VM, Martinez Zazo A, Moreno Villares JM (2010) Current status of pediatric home enteral nutrition in Spain: the importance of the NEPAD register. Nutr Hosp 25(5):810-813

8. Hebuterne X, Bozzetti F, Moreno Villares JM, Pertkiewicz M, Shaffer J, Staun M, Thul P, Van Gossum A (2003) Home enteral nutrition in adults: a European multicentre survey. Clin Nutr 22 (3):261-266

9. Howard L, Ament M, Fleming CR, Shike M, Steiger E (1995) Current use and clinical outcome of home parenteral and enteral nutrition therapies in the United States. Gastroenterology 109 (2):355-365

10. Konstan MW, VanDevanter DR, Rasouliyan L, Pasta DJ, Yegin A, Morgan WJ, Wagener JS (2010) Trends in the use of routine therapies in cystic fibrosis: 1995-2005. Pediatr Pulmonol 45 (12):1167-1172

11. Lochs H, Allison SP, Meier R, Pirlich M, Kondrup J, Schneider S, van den Berghe G, Pichard C (2006) Introductory to the ESPEN Guidelines on Enteral Nutrition: terminology, definitions and general topics. Clin Nutr 25(2):180-186 
12. Matel JL, Milla CE (2009) Nutrition in cystic fibrosis. Semin Respir Crit Care Med 30(5):579-586

13. Moreno JM, Shaffer J, Staun M, Hebuterne X, Bozzetti F, Pertkiewicz M, Thul P, Van Gossum A (2001) Survey on legislation and funding of home artificial nutrition in different European countries. Clin Nutr 20(2):117-123

14. Paccagnella A, Baruffi C, Pizzolato D, Favaro V, Marcon ML, Morello M, Semenzin M, Rebuffi S, Fossa E, Faronato P, Spinella N, Tessarin M, Foscolo G (2008) Home enteral nutrition in adults: a five-year (2001-2005) epidemiological analysis. Clin Nutr 27(3):378-385

15. Pironi L, Candusso M, Biondo A, Bosco A, Castaldi P, Contaldo F, Finocchiaro E, Giannoni A, Mazzuoli S, Orlandoni P, Palozzo A,
Panella C, Pasto S, Ruggeri E, Sandri G, Stella E, Toigo G (2007) Prevalence of home artificial nutrition in Italy in 2005: a survey by the Italian Society for Parenteral and Enteral Nutrition (SINPE). Clin Nutr 26(1):123-132

16. Truby H, Cowlishaw P, O'Neil C, Wainwright C (2009) The long term efficacy of gastrostomy feeding in children with cystic fibrosis on anthropometric markers of nutritonal status and pulmonary function. Open Respir Med J 3:112-115

17. Van Biervliet S, De Waele K, Van Winckel M, Robberecht E (2004) Percutaneous endoscopic gastrostomy in cystic fibrosis: patient acceptance and effect of overnight tube feeding on nutritional status. Acta Gastroenterol Belg 67(3):241-244 